\title{
Nuclear suppression and elliptic flow of heavy flavour decay muons at 2.76 ATeV
}

\author{
Umme Jamil ${ }^{* \dagger}$ \\ Debraj Roy College, Golaghat, Assam \\ E-mail: u_jamil@debrajroycollege.org
}

\section{Raktim Abir}

Wayne State University, Detroit, Michigan

E-mail: raktimabir@gmail.com

\section{Somnath De}

Variable Energy Cyclotron Centre, Kolkata

E-mail: somvecc@gmail.com

\section{Munshi Golam Mustafa}

Saha Institute of Nuclear Physics, Kolkata

E-mail: munshigolam.mustafaesaha.ac.in

\section{Dinesh K. Srivastava}

Variable Energy Cyclotron Centre, Kolkata

E-mail: dinesh@vecc.gov.in

\begin{abstract}
We calculate the nuclear modification factor, $R_{A A}$ and elliptic flow, $v_{2}$ of muons from heavy flavours decay at forward rapidities in $\mathrm{Pb}+\mathrm{Pb}$ collision at $\mathrm{LHC}$ and FCC energies. The $p_{T}$ distribution of heavy quarks produced from the initial fusion of partons is obtained using FONLL approach. We consider both the radiative and collision energy loss along with a boost-invariant expansion of the plasma for the prediction of $R_{A A}$ as well as $v_{2}$. We compare our result of muon $R_{A A}$ and $v_{2}$ in $\mathrm{Pb}+\mathrm{Pb}$ collision at $2.76 \mathrm{ATeV}$ with the ALICE data.
\end{abstract}

7th International Conference on Physics and Astrophysics of Quark Gluon Plasma

1-5 February, 2015

Kolkata, India

*Speaker.

${ }^{\dagger}$ A footnote may follow. 


\section{Introduction}

Heavy quarks are produced from the initial fusion of gluons or light quarks mainly during the early stage of collision. Their large mass ensures that their production can be treated using $\mathrm{PQCD}$ and also nearly negligible production at later times. While traversing the QGP they would not deviate much from the initial direction of production. So, they stands out in the sea of light quarks and gluons, which makes them an excellent probe for QGP. While passing through the QGP, they loose energy by colliding with quarks and gluons and also by radiating gluons before appearing as charm or bottom mesons or baryons. These mesons further decay through leptonic channel and thus the final spectra for these leptons would carry information about the energy loss suffered by the heavy quarks.

In this work we calculate the $p_{T}$ distribution of heavy quarks from initial fusion and then calculate the final $p_{T}$ distribution taking into account the energy loss suffered by them as they pass through the QGP. Finally we perform a Monte Carlo calculation to obtain the average change in the transverse momentum spectra of heavy quarks for nucleus-nucleus collisions and get $R_{A A}$ and $v_{2}$ as a function of $p_{T}$ for different rapidities at different centrality bins.

\section{Initial conditions and energy loss formalisms}

The $p_{T}$ distribution of heavy quarks produced from the initial fusion of partons in nucleusnucleus collisions at different centre of mass energies are obtained by Fixed Order Next to Leading Logarithm (FONLL) calculation [1]. The $p_{T}$ distribution of initial production of charm and bottom quarks at 2.76, 5.5 and $39 \mathrm{ATeV}$ obtained using FONLL calculation in $\mathrm{Pb}+\mathrm{Pb}$ collision at rapidity 2.5 are presented in Fig. 1. Here we use CTEQ 6.6 structure function set for nucleons. The Peterson fragmentation function [2] with parameter $\varepsilon_{c}=0.06$ and $\varepsilon_{b}=0.006$ are used for fragmentation of $\mathrm{c}$ quarks into D-mesons and b quarks into B-mesons, respectively. The central particle rapidity densities for $\mathrm{Pb}+\mathrm{Pb}$ collisions at 2.76, 5.5 and $39 \mathrm{ATeV}$ are taken as 2850, 3600 and 6480 respectively [3,4].

The energy loss suffered by the heavy quarks will depend upon the path-length of the heavy quarks in the plasma, the temperature evolution of the plasma, and the energy and mass of the heavy quarks. In our simple approach we make several simplifying assumptions. The heavy quarks are expected to loose most of their energy when the temperature is still large, i.e. during the earliest times after the formation of QGP, so, we can neglect the transverse expansion of the plasma. We consider Bjorken cooling works locally at different rapidities and assume a boost-invariant expansion along with a local fluid approximation.

As the heavy quarks loose most of their energy in interaction with gluon we consider only the distribution of gluons and assume a Gaussian rapidity density distribution of gluons given by $[5,6]$ :

$$
\frac{d N_{g}}{d y}=\left(\frac{d N_{g}}{d y}\right)_{0} \exp \left(-y^{2} / 2 \sigma^{2}\right) \text {. }
$$

Their density at the time $\tau$ can be written as [7]:

$$
\rho(\tau)=\frac{1}{\pi \mathrm{R}^{2} \tau} \frac{d N_{g}}{d y}
$$




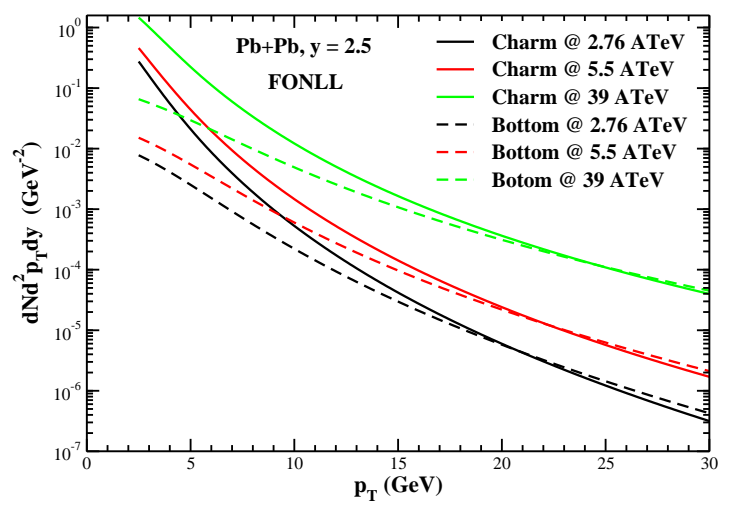

Figure 1: Initial distribution of production of charm and bottom quarks in $\mathrm{Pb}+\mathrm{Pb}$ collision at 2.76, 5.5 and $39 \mathrm{ATeV}$ at the forward rapidity 2.5 .

The corresponding temperature, assuming a chemically equilibrated plasma is

$$
T(\tau)=\left(\frac{\pi^{2}}{1.202} \frac{\rho(\tau)}{\left(9 N_{f}+16\right)}\right)^{\frac{1}{3}}
$$

In non-central collisions the heavy quarks produced from the initial fusion would cover different path lengths, depending upon the azimuthal angle and the impact parameter, inside the plasma. In a non- central collision, therefore, they would lose different amounts of energy, which will lead to an azimuthal anisotropy of momentum distribution of leptons.

A heavy quark after production at the point $(x, y)$, moves at an angle $\phi$ with respect to the reaction plane with impact parameter $\mathrm{b}$. It traverses the distance $1(\mathrm{x}, \mathrm{y}, \phi, \mathrm{b})$ inside the plasma. Using a simple approach, based on Glauber model, we calculate the dependence of the average pathlength on the azimuthal angle and impact parameter with respect to the reaction plane. Assuming uniform densities for the colliding nuclei, the average path-length for an impact parameter $\mathrm{b}$ and azimuthal angle $\phi$ can be written as [8]:

$$
\langle L(\phi ; b)\rangle=\frac{\iint l(x, y, \phi, b) T_{A B}(x, y ; b) \mathrm{d} x \mathrm{~d} y}{\iint T_{A B}(x, y ; b) \mathrm{d} x \mathrm{~d} y} .
$$

Where $T_{A B}(x, y ; b)=T_{A}(x+b / 2, y) T_{A}(x-b / 2, y)$ is the nuclear overlap function and $t_{A}$ and $t_{B}$ are the transverse density profiles of the two nuclei. An average of $L(\phi ; b)$ over $\phi$ (varying from zero to $2 \pi$ ) gives the average path length $\mathrm{L}(\phi)$ (Fig. 2).

The initial time of formation of QGP, $\tau_{0}$ is taken as $0.2 \mathrm{fm} / \mathrm{c}$. We approximate the expanding and cooling plasma with one at a temperature of $\mathrm{T}(\tau)$ at $\tau=\langle\mathrm{L}\rangle_{\mathrm{eff}} / 2$, where $\langle\mathrm{L}\rangle_{\mathrm{eff}}=\min$ $\left[\langle\mathrm{L}\rangle, v_{T} \times \tau_{c}\right]$, where $v_{T}$ is the transverse velocity of the heavy quark and $\tau_{c}$ is the critical temperature [9]. 


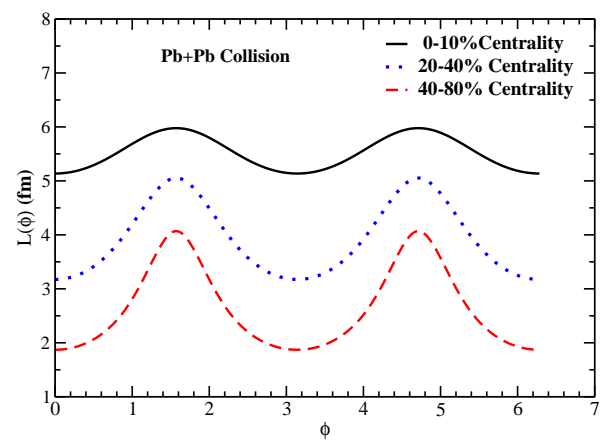

Figure 2: Variation of average path length $\mathrm{L}(\phi)$ with azimuthal angle $\phi$

The collisional energy loss suffered by heavy quarks is calculated using Peigne and Peshier (PP) [10] formalism and the radiative energy loss is calculated using AJMS [11] and DGLV formalisms [7]. We plot the transverse radiative energy loss of charm and bottom quarks, $\Delta E_{T}$ as a
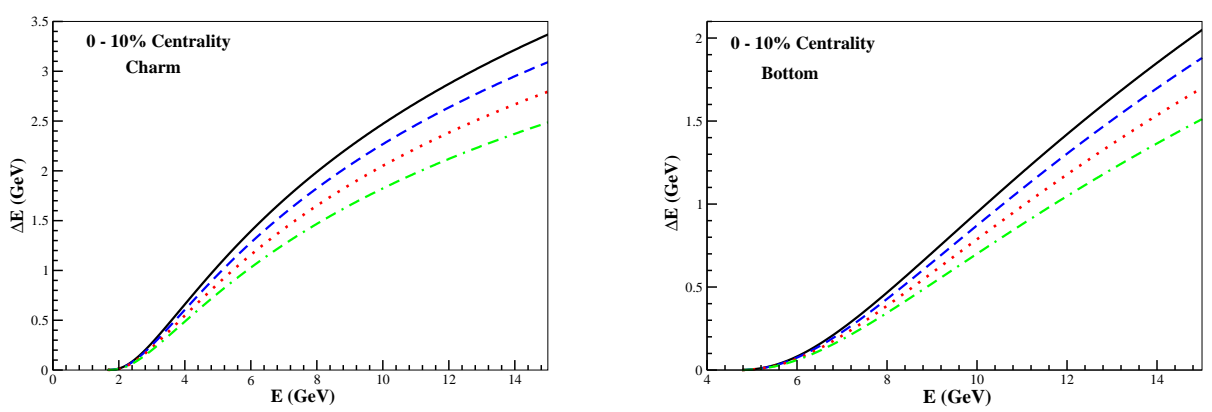

Figure 3: Radiative energy loss suffered by charm and bottom quarks while passing through the QGP at forward rapidities at $0-10 \%$ centrality.

function of transverse energy $E_{T}\left(\sqrt{p_{T}^{2}+M^{2}}\right)$ at $0-10 \%$ centrality using AJMS formalism at 2.76 $\mathrm{ATeV}$ at LHC. Each of the plots of Fig. 3 is presented for rapidities 2.5 to 4 .

\section{$R_{A A}$ of muons}

We plot the result of nuclear modification factor of muons at $0-10 \%$ centrality with both collisional and radiative energy loss at $2.76 \mathrm{ATeV}$ at LHC in Fig. 4. Our result at $0-10 \%$ centrality with only AJMS formalism found to agree well with the recent data [12] from ALICE collaboration. However, the inclusion of the collisional energy loss further suppresses the nuclear modification factor significantly and predicts more suppression compared to the experimental result. For a better understanding of the predicted trend by AJMS formalism, we also compare our results of $R_{A A}$ at 2.76, 5.5 and $39 \mathrm{ATeV}$. We see that the nuclear suppression is more with the increase of centre of 
mass energy. A detailed study comparing the predictions of $R_{A A}$ by DGLV and AJMS formalisms can be found in reference [11].
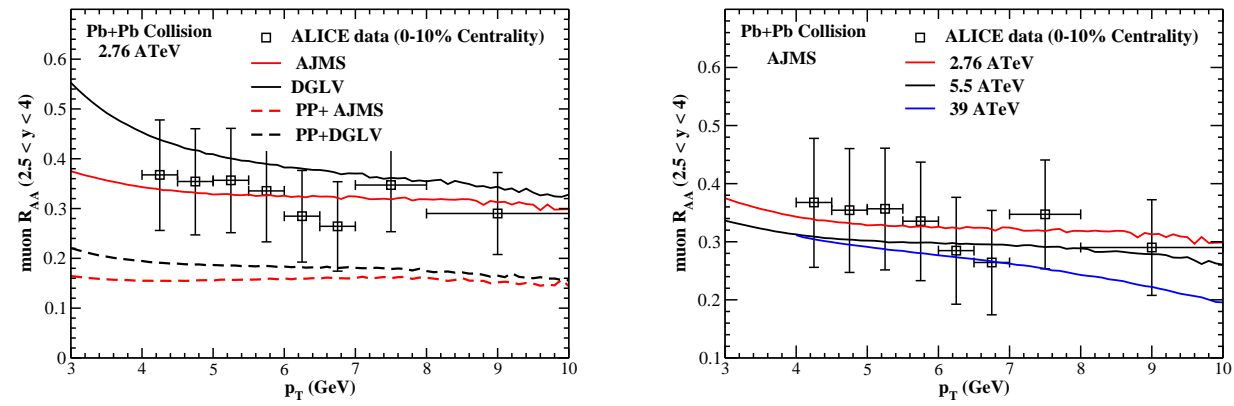

Figure 4: $R_{A A}$ of muons at forward rapidity at $0-10 \%$ centrality.
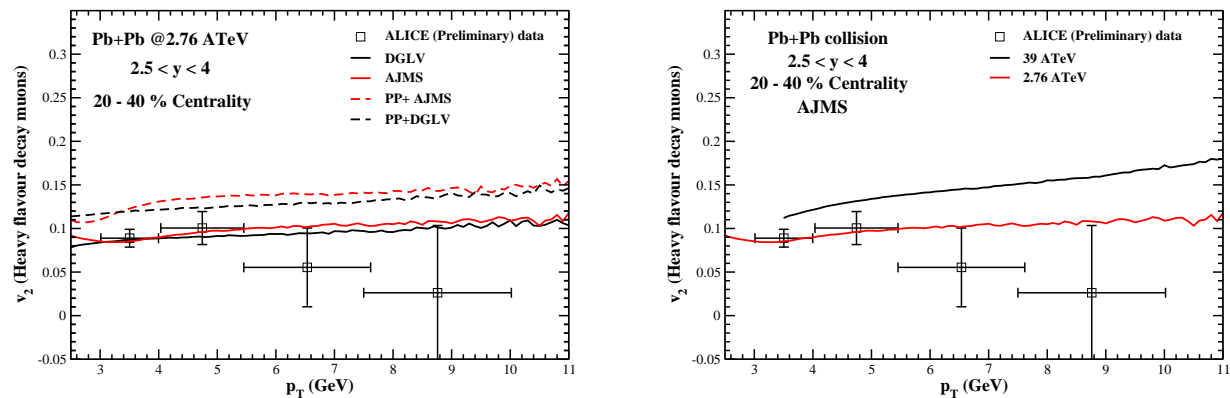

Figure 5: $v_{2}$ of muons at $2.76 \mathrm{ATeV}$ for $20-40 \%$ centrality.

\section{Azimuthal anisotropy}

We calculate the differential azimuthal anisotropy in terms of the parameter $v_{2}\left(p_{T}\right)$, which is given by:

$$
v_{2}\left(p_{T}\right)=\frac{\int_{0}^{2 \pi} \mathrm{d} \phi \cos (2 \phi) d N / d^{2} p_{T} d y}{\int_{0}^{2 \pi} \mathrm{d} \phi d N / d^{2} p_{T} d y} .
$$

In Fig. 5 we plot the result of azimuthal anisotropy of muons in $\mathrm{Pb}+\mathrm{Pb}$ collision at $2.76 \mathrm{ATeV}$ for $20-40 \%$ centrality in the rapidity window 2.5 to 4 . We compare the predicted result with the preliminary ALICE data of azimuthal anisotropy of heavy flavour decay muons [13]. We also compare our prediction of $v_{2}\left(p_{T}\right)$ at 2.76 and $39 \mathrm{ATeV}$ in the same rapidity window with AJMS 
formalism. From these comparisons, we feel that we need more experimental data for $v_{2}\left(p_{T}\right)$ at high $p_{T}$ to understand our predicted trend.

\section{Summary}

Our prediction of $R_{A A}$ considering only radiative energy loss agrees well with the ALICE data, but when we consider both the radiative and collisional energy losses, it shows more suppression than the experimental data. In our model we are predicting maximum possible energy loss by assuming one dimensional expansion of the plasma and also by considering constant density distribution of the colliding nuclei while calculating the average path length traversed by the quark. In turns we are predicting maximum possible nuclear suppression and azimuthal anisotropy. We expect that the scenario can be improved by incorporating transverse expansion of the plasma along with Wood-saxon density distribution for the colliding nuclei.

\section{References}

[1] M. Cacciari et al., J. High Energy Phys. 9805 (1998) 007; J. High Energy Phys. 0103 (2001) 006.

[2] C. Peterson et al., Phys. Rev. D 27 (1983) 105.

[3] K. Aamdot, et al., [ALICE Coll], Phys. Rev. Lett. 105 (2010) 252301.

[4] N. Armesto et al., Nucl. Phys. A 00 (2014) 1.

[5] R. Vogt, B. V. Jacak, P. L. McGaughey, and P. V. Ruuskanen, Phys. Rev. D 49, 3345 (1994).

[6] S. Gavin, P. L. McGaughey, P. V. Ruuskanen, and R. Vogt, Phys. Rev. C 54, 2606 (1996).

[7] M. Djordjevic and M. Gyulassy Nucl. Phys. A 733, 265 (2004); S. Wicks et al., Nucl. Phys. A 784, 426 (2007).

[8] S. De, D. K. Srivastava J. Phys. G 39 (2012) 015001.

[9] U. Jamil and D. K. Srivastava, J. Phys. G 37, 085106 (2010).

[10] S. Peigne and A. Peshier, Phys. Rev. D 77, 114017 (2008).

[11] R. Abir et al., Phys. Lett. B 715, 183 (2012); arXiv:1203.5221 (2012).

[12] ALICE Collaboration, Phys. Rev. Lett. 109, 112301 (2012).

[13] Grazia Luparello for the ALICE Collaboration, arXiv:1411.2442v1 (2014). 